Original Article

\title{
$\beta$-Sitosterol treatment attenuates cognitive deficits and prevents amyloid plaque deposition in amyloid protein precursor/ presenilin 1 mice
}

\author{
Jian-Ya Ye ${ }^{1}$, Li Li ', Qing-Mao Hao ${ }^{1}$, Yong Qin ${ }^{1}$, and Chang-Sheng $\mathrm{Ma}^{2 \text {,* }}$ \\ ${ }^{1}$ Hebei University of Chinese Medicine, Shijiazhang 050200, ${ }^{2}$ Neurobiology Laboratory, Institute of Basic Medicine, Hebei Medical University, Shijiazhang \\ 050017, Hebei province, China
}

\section{ARTICLE INFO}

Received June 29, 2019

Revised July 23, 2019

Accepted August 5, 2019

*Correspondence

Chang-Sheng Ma

E-mail: machangsheng0311@163.com

Key Words

Alzheimer disease

Amyloid-beta

$\beta$-Sitosterol

Learning and memory
ABSTRACT Alzheimer's disease (AD) is the most common neurodegenerative disorder causing dementia worldwide, and is mainly characterized by aggregated $\beta$-amyloid $(A \beta)$. Increasing evidence has shown that plant extracts have the potential to delay $A D$ development. The plant sterol $\beta$-Sitosterol has a potential role in inhibiting the production of platelet $A \beta$, suggesting that it may be useful for AD prevention. In the present study, we aimed to investigate the effect and mechanism of $\beta$-Sitosterol on deficits in learning and memory in amyloid protein precursor/presenilin 1 (APP/PS1) double transgenic mice. APP/PS1 mice were treated with $\beta$-Sitosterol for four weeks, from the age of seven months. Brain A $\beta$ metabolism was evaluated using ELISA and Western blotting. We found that $\beta$-Sitosterol treatment can improve spatial learning and recognition memory ability, and reduce plaque load in APP/PS1 mice. $\beta$-Sitosterol treatment helped reverse dendritic spine loss in APP/PS1 mice and reversed the decreased hippocampal neuron miniature excitatory postsynaptic current frequency. Our research helps to explain and support the neuroprotective effect of $\beta$-Sitosterol, which may offer a novel pharmaceutical agent for the treatment of AD. Taken together, these findings suggest that $\beta$-Sitosterol ameliorates memory and learning impairment in APP/PS1 mice and possibly decreases $A \beta$ deposition.

\section{INTRODUCTION}

As a progressive neurodegenerative disease, Alzheimer's disease (AD) is the most common form of dementia worldwide, affecting more than 30 million people worldwide [1]. The main pathological characteristics of $\mathrm{AD}$ are extracellular amyloid $\beta(\mathrm{A} \beta)$ plaque deposition and neurofibrillary tangles (NFT) [2]. A $\beta$ is derived from the amyloid protein precursor (APP) and NFT are mainly composed of hyperphosphorylated tau [3]. The production and clearance rates of $\mathrm{A} \beta$ maintain a dynamic balance in normal individuals [4]. The imbalance between the production and removal of $A \beta$ results in the formation of $A \beta$ oligomers, which eventually accumulate into senile plaques in the brain [3]. The accumula- tion of this peptide in $\mathrm{AD}$ patients leads to synaptic dysfunction, inflammation stress, apoptosis of neurons and cognitive deficits $[5,6]$. Significant progress has been made in therapies for $A D$ in recent years; immunotherapy, targeting $A \beta$, is considered to be the most promising treatment due to its specific activity to limit the production, accumulation and deposition of $A \beta$ [7]. However, serious side effects limit wide clinical application. There is an urgent need for complementary therapies, such as naturally derived drugs.

Drugs made from plant compounds have important clinical potential for the treatment of AD [8]. Natural compounds extracted from plants, such as catechins [9] and curcumin [10], have shown beneficial effects in $\mathrm{AD}$. As a compound with a structure
(1) $\$$ This is an Open Access article distributed under the terms of the Creative Commons Attribution Non-Commercial License, which permits unrestricted non-commercial use, distribution, and reproduction in any medium, provided the original work is properly cited. Copyright @ Korean J Physiol Pharmacol, pISSN 1226-4512, eISSN 2093-3827
Author contributions: J.Y.Y. conceived the experiments, L.L. contributed to research data, J.Y.Y. drafted the manuscript; Q.M.H. contributed to technical assistance; Y.Q. participated in raising animals; C.S.M. revised the manuscript and supervised the analysis. 
similar to cholesterol, $\beta$-Sitosterol is one of the most widely distributed plant sterols existing in plants [11]. $\beta$-Sitosterol has been widely investigated in medical research [12]. Dietary phytosterols can accumulate in brain tissue via the blood-brain barrier (BBB) and may affect brain function [13]. $\beta$-Sitosterol changes the shear pattern of APP [14], suggesting that it may be a potential therapy for $\mathrm{AD}$. However, whether it has a neuroprotective effect on $\mathrm{AD}$ in vivo is still unclear. APP/presenilin 1 (PS1) transgenic mice show spatial memory impairment and imbalanced amyloid production and clearance $[15,16]$. In the present study, we simulated the pathogenesis of $\mathrm{AD}$ in APP/PS1 mice and used $\beta$-Sitosterol as an intervention method, to explore the effect and mechanism on cognitive function in these mice. We report, for the first time, that administration of $\beta$-Sitosterol in APP/PS1 mice can reduce $\mathrm{A} \beta$ deposition and improve cognitive impairment. These results strongly support that $\beta$-Sitosterol is a promising therapeutic agent for AD.

\section{METHODS}

\section{Animals and drug administration}

Age matched, gender mixed APP/PS1 and wild-type (WT) littermates were purchased from Shanghai Nanfang Research Center for Model Organisms (Shanghai, China). Mice were housed no more than five per cage, with free access to food and water, under standard specific-pathogen-free laboratory conditions. The mice were handled to adapt to the experimenter before behavior testing. All animal procedures were performed in strict accordance with the guidelines for the care and use of laboratory animals of the Institute for Experimental Animals at Hebei Medical University and with the laws of China. All experiments were performed in accordance with the guidelines for Animal Care and Use of China, and the experimental schemes were approved by the animal ethics committee of Hebei Medical University (approval No. 2018011201A). The 30-week-old mice were divided into three groups: WT littermate controls (WT), APP/PS1 vehicle-treated controls (APP/PS1) and APP/PS1 treated with $\beta$-Sitosterol (APP/ PS1 + BSS). $\beta$-Sitosterol was purchased from Sigma-Aldrich (Sigma, St. Louis, MO, USA) and dissolved in dimethyl sulfoxide (DMSO) as a stock solution $(20 \mathrm{mg} / \mathrm{kg})$. Control groups were treated with $0.1 \%$ DMSO. All groups were treated once per day by gavage for continuously 28 days.

\section{Behavioral testing}

Three days after $\beta$-Sitosterol administration, spatial learning and recognition memory testing was performed during the daylight hours.

The Morris water maze (MWM) test was conducted following a previously described method [17]. Briefly, A stainless steel pool
(110 $\mathrm{cm}$ in diameter) was divided into four quadrants, containing a submerged escape platform $(10 \mathrm{~cm}$ in diameter) located $1 \mathrm{~cm}$ under the water surface. The water temperature was maintained at $23 \pm 1^{\circ} \mathrm{C}$. The acquisition period consisted of 5 consecutive days of training with four trials/day. The time taken for the mouse to swim onto the hidden platform was recorded for all mice. Each mouse was allowed up to $60 \mathrm{sec}$ to find the hidden platforms. If a mouse could not find a platform within $60 \mathrm{sec}$, the training was interrupted and the mouse was guided to a platform, to remain for $10 \mathrm{sec}$. The time that mice spent in the target quadrant and the number of target platform crossings, were recorded. On the 6 th day, the platform was removed and spatial probe trials were conducted. Mice were given $60 \mathrm{sec}$ to perform their search bias.

The novel object recognition test was conducted in a self-made open-field arena $(58 \times 58 \times 31 \mathrm{~cm})$ as previously described [18]. Mice were placed in the arena for $10 \mathrm{~min}, 24 \mathrm{~h}$ prior to the test, to acclimatize them to the apparatus and environment. Seventy percent ethanol was used to remove olfactory cues. Exploratory objects were red cubes $(1.5 \times 1.5 \times 1.5 \mathrm{~cm})$ or white cubes $(2 \times 2 \times$ $2 \mathrm{~cm}$ ) and mice were allowed $10 \mathrm{~min}$ for exploration. After a 3-h retention interval, mice were returned to the arena and exposed to the objects, in addition to a novel object, for a 10-min recognition trial. Object location and type were randomized throughout the trial. Total time spent exploring the familiar objects (TF $=$ time spent exploring the familiar object) or the novel object (TN $=$ time spent exploring the novel object) was recorded by SMART 3.0 software (Panlab, Barcelona, Spain). A recognition index was used to measure recognition memory: [TF or TN / $(\mathrm{TF}+\mathrm{TN})] \times$ 100. The discrimination index could be get by TN minus TF. All behavioral parameters of mice were tracked, recorded and analyzed using SMART ver. 3.0 (Harvard Apparatus, Holliston, MA, USA).

\section{ELISA}

Brain tissue was homogenized and supernatant samples containing soluble $A \beta$, or pellets containing insoluble $A \beta$, were collected. The hippocampus and cortex A $\beta 1-40$ and $A \beta 1-42$ levels were measured by ELISA according to the manufacturer's instructions (ELISA; BioSource International, Camarillo, CA, USA). The values of $A \beta$ concentrations were acquired with reference to the standard curve.

\section{Thioflavin S staining}

Four percent paraformaldehyde ( $\mathrm{pH}$ 7.4) fixed brain tissues were collected and dehydrated in $30 \%$ sucrose, and $35 \mu \mathrm{m}$ frozen section slices were used for Thioflavin S (ThS) (1\%, 8 min) staining. Coverslips were mounted using depex polystyrene (Electron Microscopy Sciences, Hatfield, PA, USA). Hippocampal images were taken with a Nikon digital camera (Coolpix 4500; Nikon, Tokyo, Japan). The A $\beta$ plaques were counted in five representative 
sections from each animal.

\section{Western blotting}

Protein extraction from the hippocampus was performed as previously described [19]. Protein concentration was determined using the BCA protein assay (Tiangen Biotech Co. Ltd., Beijing, China). $10 \mu \mathrm{g}$ of protein from each sample was separated by $10 \%$ SDS-PAGE and electrotransferred to polyvinylidene fluoride membranes (Millipore, Billerica, MA, USA) for immunoblotting. The following primary antibodies were used: anti-A $\beta$ (6E10, 1:1000; Covance, Emeryville, CA, USA), anti-BACE1 (1:500, ab2077; Abcam, Cambridge, MA, USA), and anti- $\beta$-Actin (1:500, ab2077; Abcam). Band intensities were semi-quantified using ImageJ ver. 1.6 (http://imagej.nih.gov/ij/) software.

\section{Dendritic spine density analysis}

$200 \mathrm{nl}$ AAV-IRES-GFP $(2.45 \mathrm{E}+13 \mathrm{vg} / \mathrm{ml}$; OBiO, Shanghai, China) viral vector were stereotactically injected in the hippocampus to label the DG neurons. Fourteen days after the infec- tion, the mice were perfused and brain were extracted. Forty $\mu \mathrm{m}$ frozen sections were used for quantification of dendritic spines which was evaluated by fluorescent confocal imaging (LSM 510; Carl Zeiss, Jena, Germany) with identical settings for all samples. Spines were counted on 30-40 $\mu \mathrm{m}$ segments of secondary dendrites extending at least $60 \mu \mathrm{m}$ beyond the cell body. Five segments from each neuron were quantified.

\section{Whole-cell patch clamp studies}

Whole cell voltage-clamp was performed at $37^{\circ} \mathrm{C}$ with a 60-channel voltage amplifier (MEA1060-Inv-BC; Multi-Channel Systems, Reutlingen, Germany). All recordings were monitored in all experiments. Simultaneous whole-cell current and voltage clamps were held at $-68 \mathrm{mV}$ during recording. Patch pipettes had an identical resistance of 3-4M $\Omega$. The frequency and amplitude of the miniature excitatory postsynaptic currents (mEPSC) were sampled at $10 \mathrm{kHz}$ and low-pass filtered at $3 \mathrm{kHz}$ (Axon Digidata 1440A; Molecular Devices, Union City, CA, USA). The noise level was below $4 \mathrm{pA}$, and $7 \mathrm{pA}$ and was typically used as the threshold for mEPSC events. Five min of representative mEPSC recordings

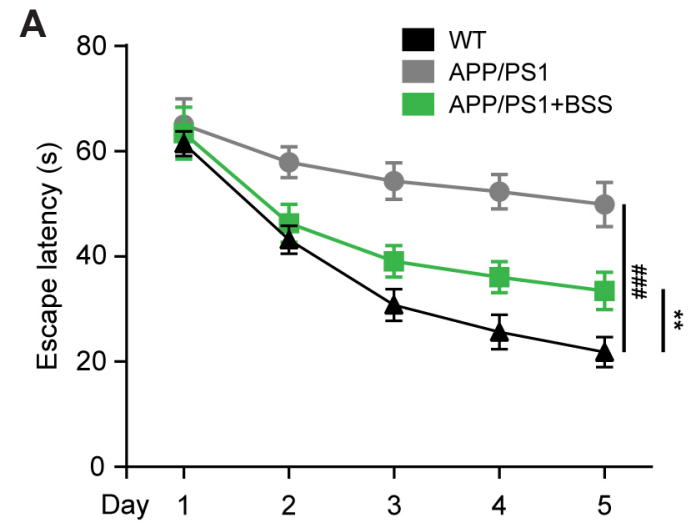

D

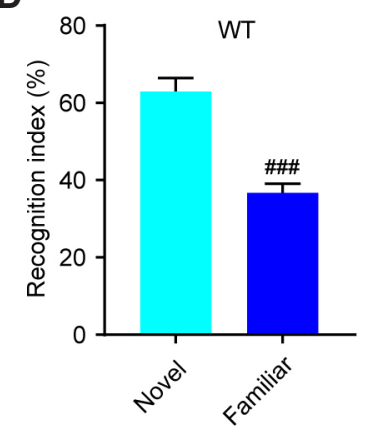

B

E

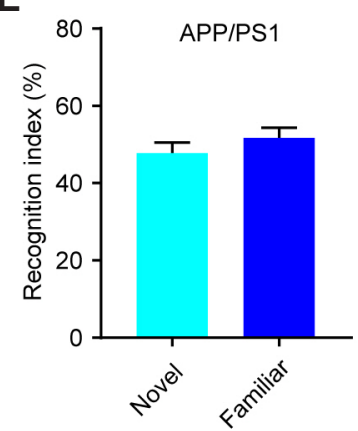

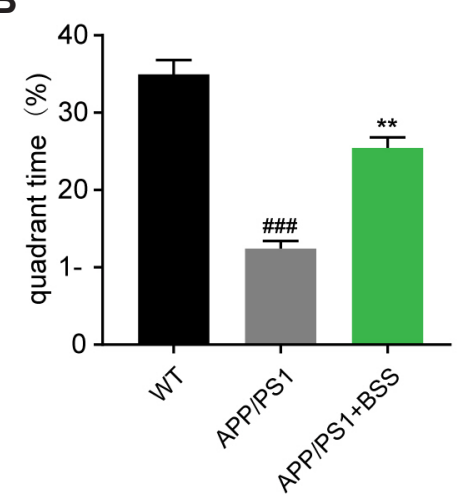

$\mathbf{F}$

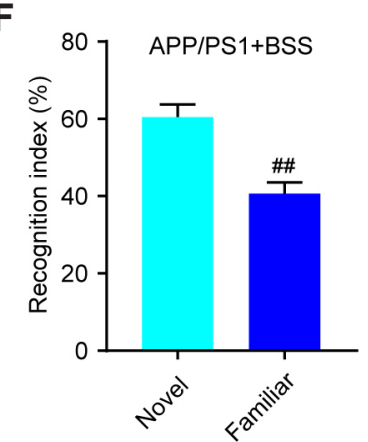

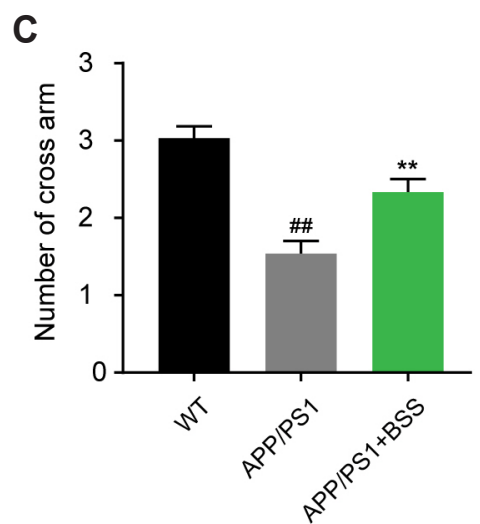

G

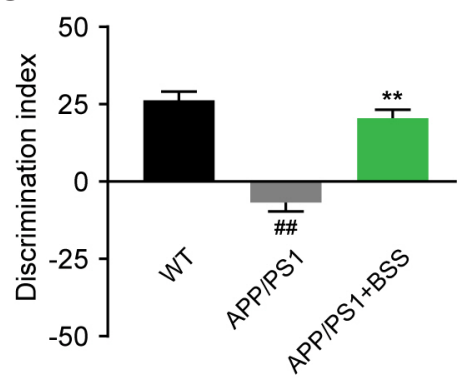

Fig. 1. $\beta$-Sitosterol treatment attenuates spatial learning and recognition memory deficits in amyloid protein precursor/presenilin 1 (APP/ PS1) mice. (A) The escape latency of APP/PS1 mice in the navigation test. (B) The percentage of time spent in the target quadrant of APP/PS1 mice in the probe test. (C) The number of target platform crossings of APP/PS1 mice in the probe test. (D) Recognition preference for novel object of wild-type (WT) mice. (E) Recognition preference for novel object in control APP/PS1 mice. (F) Recognition preference for novel object in $\beta$-Sitosterol treated APP/PS1 mice. (G) Statistical results of recognition difference scores for novel object in $\beta$-Sitosterol treated APP/PS1 mice. Data are presented as mean \pm standard error of the mean, $\mathrm{n}=10-12$ mice per group, gender mixed. One-way ANOVA, Bonferroni multiple comparisons test or Student's t-test were used: ${ }^{\#} p<0.01, \# \#<0.001,{ }^{* *} p<0.01$. 
were used to generate the cumulative distribution plot.

\section{Statistical analyses}

All values were expressed as the mean \pm the standard error of the mean. Data management and statistical analyses were performed using GraphPad Prism ver 7.0 (GraphPad Software, Inc., San Diego, CA, USA). All statistical assessments were two-sided and the corresponding $\mathrm{p}$-values were calculated. Statistical significance was determined at the $\alpha$-level of significance of $p=0.05$. Statistical analyses were carried out according to the experimental design used and are listed in figure legends.

\section{RESULTS}

\section{$\beta$-Sitosterol treatment rescues spatial and recognition memory deficits in APP/PS1 mice}

To investigate whether $\beta$-Sitosterol treatment could attenuate hippocampus-dependent learning and memory impairment in APP/PS1 mice, age-matched WT, APP/PS1 and $\beta$-Sitosterol treated mice were assessed by the MWM and novel object recognition (NOR) tests. Our results showed that the $\beta$-Sitosterol treated APP/PS1 mice showed reduced escape latency (Fig. 1A). In the probe trial phase, the $\beta$-Sitosterol treated mice performed better than the APP/PS1 controls, as reflected by the significantly greater time spent in the target quadrant and more crossovers from the former platform (Fig. 1B, C). These observations indicate that $\beta$-Sitosterol treatment ameliorated spatial learning deficits in APP/PS1 mice. In the object recognition memory test, $\beta$-Sitosterol treated APP/PS1 mice and WT mice showed similar significant recognition of the novel object (Fig. 1D, F), but the APP/PS1 control mice did not (Fig. 1E). APP/PS1 vehicle-treated mice attained a significantly impaired score for novel object recognition (Fig. 1G).

\section{$\beta$-Sitosterol treatment rescues A $\beta$ pathology in APP/ PS1 mice}

An association has been observed between $\mathrm{A} \beta$ deposition in the brains of both Alzheimer's disease patients and transgenic mouse models. After behavioural test, hippocampal and cortical tissues were isolated from each group. Total protein was extracted and quantified by $\mathrm{BCA}$, the concentration of soluble and insoluble $\mathrm{A} \beta 40$ and $\mathrm{A} \beta 42$ in the brain was determined by using $\mathrm{A} \beta$ ELISA kit. Total soluble and insoluble brain $A \beta 42$ or $A \beta 40$ levels were significantly increased in the control APP/PS1 mice conpared with the WT control in both brain regions (Fig. 2). Insoluble tissue fractions showed the most prominent increases in A $\beta 42$ (Fig. 2). However, the $A \beta 40$ and $A \beta 42$ were markly reduced in $A P P /$ PS1 mice treated with $\beta$-Sitosterol (Fig. 2).
A

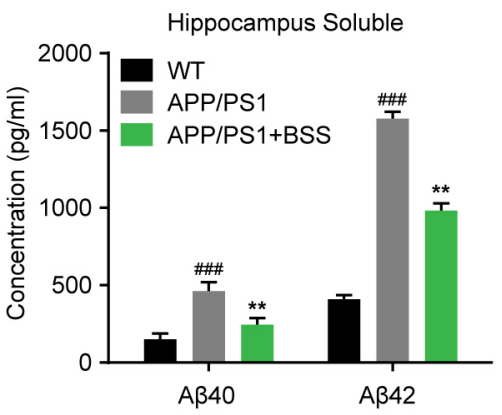

C

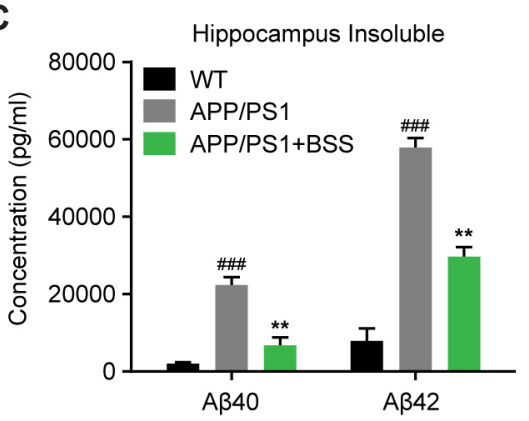

B
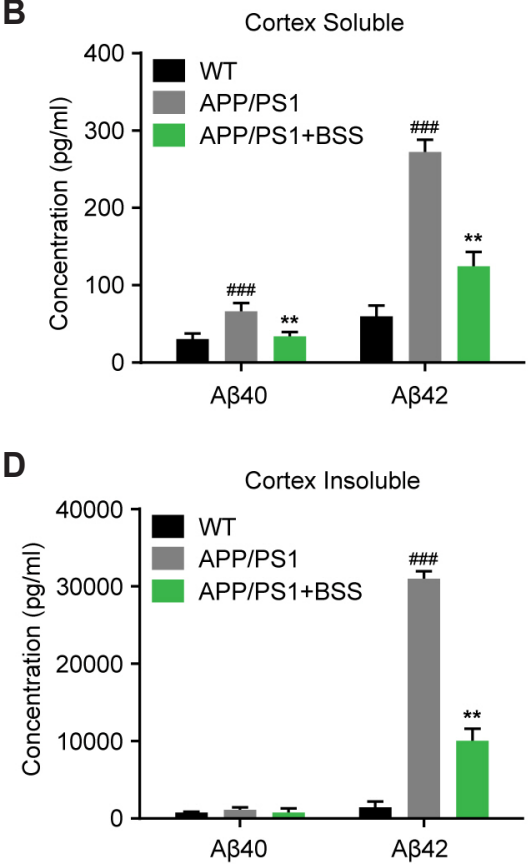

Fig. 2. Analysis of $\beta$-amyloid (A $\beta$ ) metabolism in $\beta$-Sitosterol treated amyloid protein precursor/presenilin 1 (APP/PS1) mice. (A, B) ELISA of soluble $A \beta 40$ and $A \beta 42$ in hippocampal and cortical samples of APP/PS1 mice. (C, D) ELISA of insoluble A $\beta 40$ and A $\beta 42$ in hippocampal and cortical samples of APP/PS1 mice. Data are presented as mean \pm standard error of the mean. $n=9-12$ mice per group, gender mixed. One-way ANOVA, Bonferroni multiple comparisons test or Student's t-test were used: ${ }^{\# \#} p<0.001$ vs. wild-type (WT), ${ }^{* *} p \leq 0.001$ vs. APP/PS1 mice. 


\section{$\beta$-Sitosterol treatment attenuated $A \beta$ accumulation and BACE1 expression}

We further determined $A \beta$ accumulation in the brain, by thioflavin-S staining and immunoblotting. $A \beta$ staining result showed that $\beta$-Sitosterol administration results in a decrease in $A \beta$ density in the hippocampus and cortex of APP/PS1 mice (Fig. $3 \mathrm{~A}, \mathrm{~B})$. Similarly, immunoblotting for $\mathrm{A} \beta$ and BACE1 expression showed that $\beta$-Sitosterol treatment significantly decreased their expression compared with the control APP/PS1 mice (Fig. 3C).

\section{$\beta$-Sitosterol treatment restored the synaptic dysfunction in APP/PS1 mice}

Dendritic spine and synaptic loss are well documented in APP/ PS1 mice. We used AAV-IRES-GFP to label the hippocampal neurons of APP/PS1 mice. The dendritic spine densities of hippocampal neurons were analyzed for each group. $\beta$-Sitosterol treatment caused up-regulation of dendritic spine density compared with control APP/PS1 mice (Fig. 4A, B). Next, we used a single whole-cell recording method to record mEPSCs, to quantify synaptic transmission. The mEPSC frequency of APP/PS1 mice hippocampal neurons was significantly decreased compared with WT mice. However, in mice treated with $\beta$-Sitosterol, the mEPSC frequency showed a reversal to control levels. The mEPSC amplitude was not significantly different between any of the groups (Fig. $4 \mathrm{C})$. These results showed that $\beta$-Sitosterol treatment rescued the synaptic transmission in APP/PS1 mice.

\section{DISCUSSION}

$\mathrm{AD}$ is a progressive neurodegenerative disorder characterized by $A \beta$ plaque deposits, NFT, and neuronal loss, leading to learning and memory impairments [20]. Increased $A \beta$ plaque deposition and decreased neuronal function in the hippocampus is implicated in the loss of memory performance in $\mathrm{AD}$ mice [21]. $\beta$-Sitosterol has been reported to cross the BBB and inhibit the production of $A \beta[22,23]$ and we speculate that this may contribute to a delay in the pathological development of $\mathrm{AD}$. Therefore, we treated APP/PS1 mice with $\beta$-Sitosterol and evaluated cognitive dysfunction and extent of AD pathology. The APP/ PS1 transgenetic mouse model has been widely used to explore the cognitive deficits related to AD. APP/PS1 mice show pro-

\section{A}
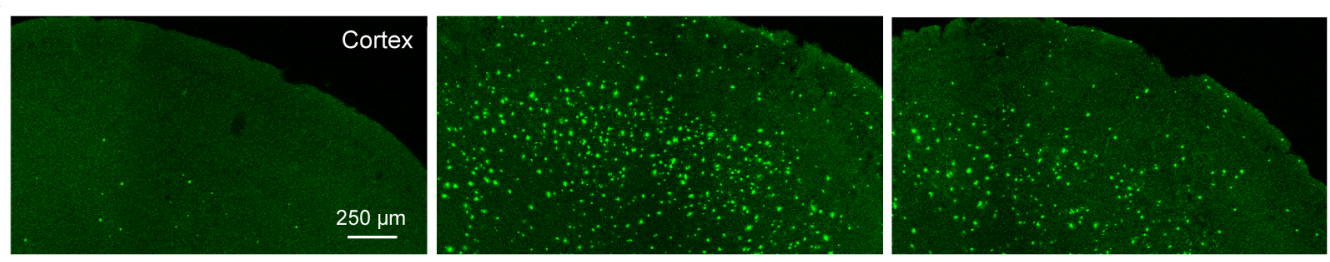

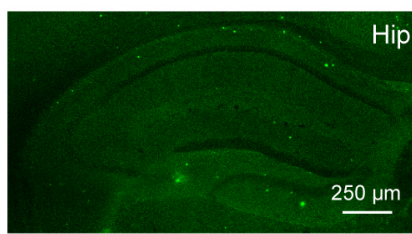

WT

B

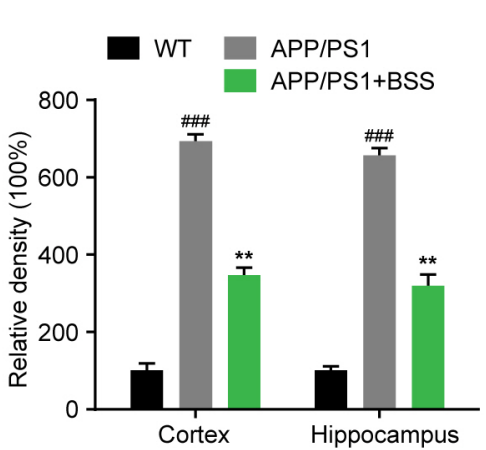

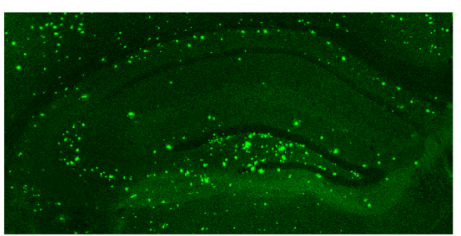

APP/PS1

C

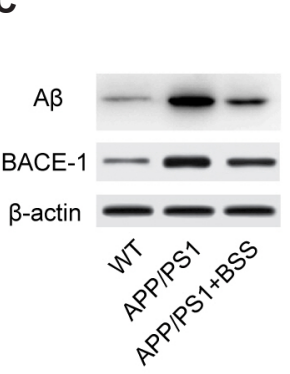

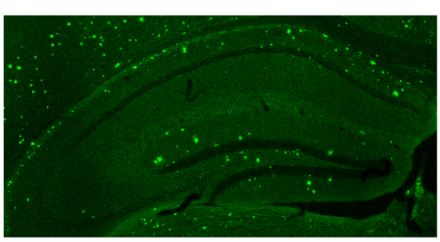

APP/PS1+BSS

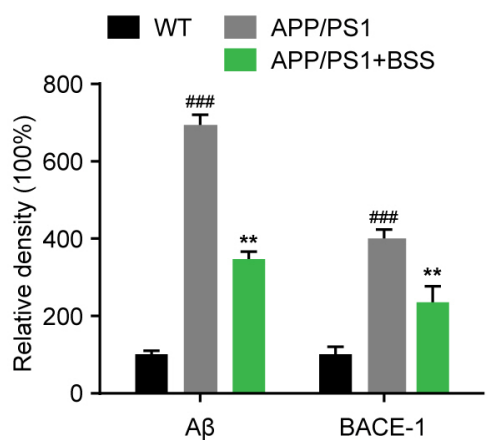

Fig. 3. $\beta$-Sitosterol treatment attenuated $\beta$-amyloid $(A \beta)$ and BACE1 protein expression in amyloid protein precursor/presenilin 1 (APP/PS1) mice. (A) A $\beta$ staining of the hippocampus and cortex of APP/PS1 mice $(\times 40)$. (B) The A $\beta$ density values are expressed in arbitrary units. (C) Immunoblot semi-quantitative analysis of A $\beta$ and BACE1 protein expression in the hippocampus of APP/PS1 mice. Data are presented as mean \pm standard error of the mean. $n=12-16$ mice per group, gender mixed. One-way ANOVA, Bonferroni multiple comparisons test or Student's $t$-test were used: ${ }^{\# \#} \mathrm{p}<0.001$ vs. wild-type (WT), ${ }^{* *} \mathrm{p} \leq 0.001$ vs. APP/PS1 mice. 

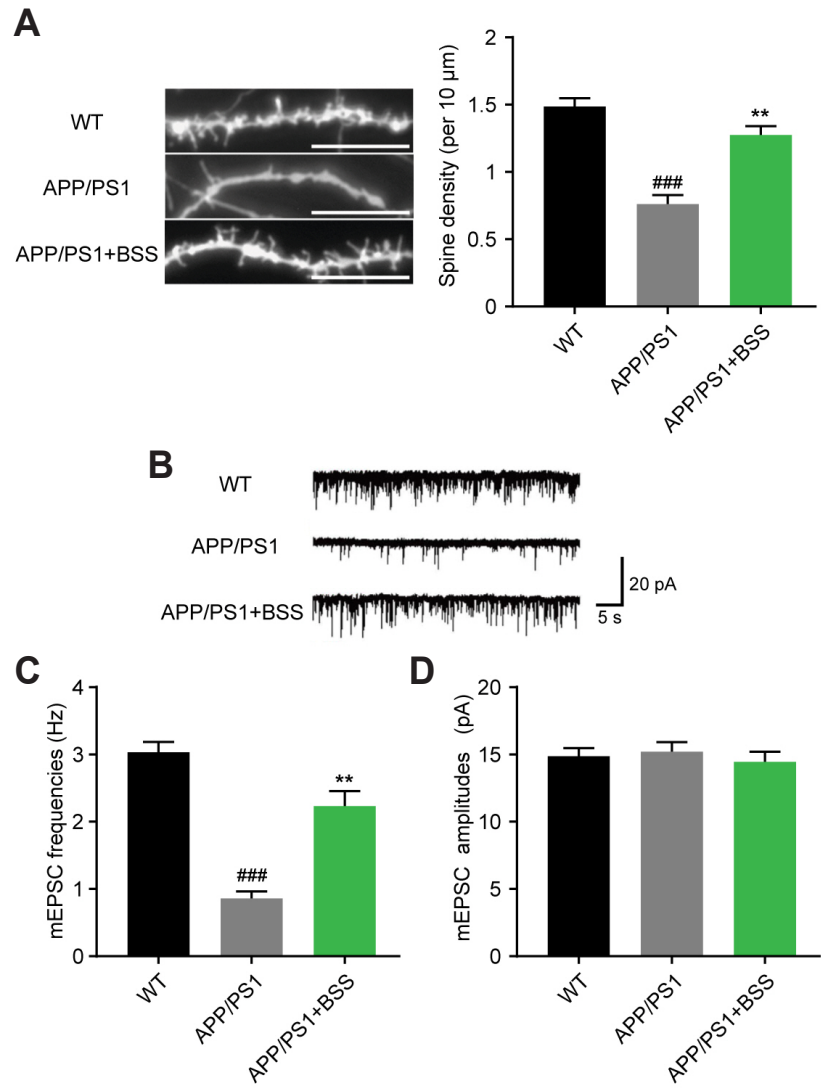

B $\quad w T$

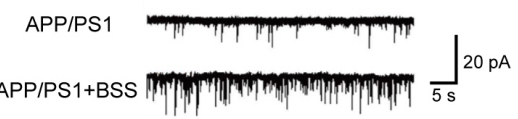

D

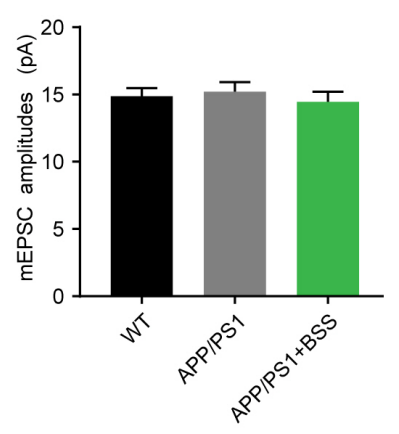

Fig. 4. $\beta$-Sitosterol treatment restored synaptic dysfunction in amyloid protein precursor/presenilin 1 (APP/PS1) mice. (A) Confocal images of green fluorescent protein-labeled-hippocampal neurons were used to visualized the dendritic spines. 6-8 field per animal, 7-8 animals per group. (B) Representative traces of miniature excitatory postsynaptic currents ( $\mathrm{mEPSCs}$ ) recorded in hippocampal neurons of each group. (C, D) Statistical analyses of the mean values of mEPSC frequencies and amplitudes in hippocampal neurons of each group. Data are presented as mean \pm standard error of the mean. $n=9-12$ mice per group, gender mixed. One-way ANOVA, Bonferroni multiple comparisons test or Student's t-test were used: ${ }^{\# \#} p<0.001$ vs. wild-type (WT), ** $\mathrm{p} \leq 0.001$ vs. APP/PS1 mice.

gressive accumulation of amyloid plaques and cognitive deficits after seven months [24]. Therefore, we initiated drug treatment in 30-week-old APP/PS1 mice, and continued for 4 weeks. We investigated the underlying role of $\beta$-Sitosterol in the protection against $\mathrm{AD}$ progression. The pathological characteristics of APP/PS1 mice, represented by memory and recognition deficits, impaired $A \beta$ metabolism and synaptic loss, were evaluated to investigate the efficacy of $\beta$-Sitosterol in alleviating AD-related pathologies. Herein, we found that APP/PS1 transgenic mice treated with $\beta$-Sitosterol had significantly better working memory and visual recognition memory in the MWM and NOR test, compared with the $\mathrm{AD}$ control group. The behavioral results showed an observable decrease in escape latency and an increase in time spent on the target platform in the MWM test, compared with vehicle treated APP/PS1 mice. We suggest that $\beta$-Sitosterol plays a protective role in cognitive dysfunction during $\mathrm{AD}$ occurrence.

Imbalanced $\mathrm{A} \beta$ metabolism is a pathological characteristic of $\mathrm{AD}$ [25]. Accumulative $\mathrm{A} \beta$ production and aggregation in the brain has been associated with neuronal dysfunction and memory disorders $[26,27]$. Inhibiting $A \beta$ production or facilitating $A \beta$ degradation are effective strategies for addressing AD-like pathologies [28]. Here, we provide in vivo evidence that $\beta$-Sitosterol is implicated in the mechanisms of $A \beta$ production and deposition in mice. We identified roles of $\beta$-Sitosterol in the regulation of $\mathrm{A} \beta$ metabolism, providing a potential mechanism for the effects observed in the behavior test. We assessed amyloid plaque metabolism and found markedly reduced amyloid plaque deposits in the hippocampus and cortex. Beta-secretase 1 serves as the initiating enzyme in APP processing and $A \beta$ production. The inhibition of BACE1 has been shown to be an effective strategy for $A \beta$ clearance. The decreased expression of BACE1 in the brains of $\beta$-Sitosterol treated APP/PS1 mice is supported by the ELISA and Western blot analysis of $A \beta$. Our findings showed that the expression of BACE1 was down-regulated in the hippocampus after treatment with $\beta$-Sitosterol. This result implies that $\beta$-Sitosterol could also affect $B A C E 1$ to reduce the generation of $A \beta$ in the $A D$ brain.

Synaptic loss is widely observed in human and mice AD brains $[29,30]$. Disconnections between neurons are responsible for the loss of cognitive function in $\mathrm{AD}$ patients. Through quantification of synapse numbers in the hippocampus, we demonstrated that $\beta$-Sitosterol treatment partly rescued the reduction in dendritic spine density in hippocampal neurons from APP/PS1 mice. In addition, the decreased frequency of mEPSCs was ameliorated. Synaptic spine density and neural electrical activity is believed to be a synaptic mechanism underlying recognition and memories in the brain [31,32]. Therefore, maintenance of hippocampal spine and neural spiking explains the improved behavioral outcomes in $\beta$-Sitosterol-treated APP/PS1 mice.

In conclusion, the results presented in this study suggest that $\beta$-Sitosterol has therapeutic effects and reduces Alzheimer's disease progression in the aged APP/PS1 mouse model, which mimics the later stages of $\mathrm{AD}$, raising hopes that $\beta$-Sitosterol may show positive effects, even in the later stages, for the AD patient.

Based on the encouraging effects that we found with $\beta$-Sitosterol, the neuroprotective effects of $\beta$-Sitosterol on other AD mouse models should also be examined, before commencement of clinical trials

Issues remaining for further study include identifying the molecular mechanisms underlying $A \beta$ clearance and accumulation, and determining whether this is due to the promotion of $A \beta$ clearance.

\section{CONFLICTS OF INTEREST}

The authors declare no conflicts of interest. 


\section{REFERENCES}

1. Aprahamian I, Stella F, Forlenza OV. New treatment strategies for Alzheimer's disease: is there a hope? Indian J Med Res. 2013;138:449460.

2. Zhang LH, Wang X, Stoltenberg M, Danscher G, Huang L, Wang ZY. Abundant expression of zinc transporters in the amyloid plaques of Alzheimer's disease brain. Brain Res Bull. 2008;77:55-60.

3. Ma SL, Pastorino L, Zhou XZ, Lu KP. Prolyl isomerase Pin1 promotes amyloid precursor protein (APP) turnover by inhibiting glycogen synthase kinase-3 $\beta$ (GSK3 $\beta$ ) activity: novel mechanism for Pin1 to protect against Alzheimer disease. J Biol Chem. 2012;287:6969-6973.

4. Bateman RJ. Amyloid-beta production and clearance rates in Alzheimer's disease. J Alzheimer's Assoc. 2010;6(4 Suppl):S101.

5. Gordon MN, King DL, Diamond DM, Jantzen PT, Boyett KV, Hope CE, Hatcher JM, DiCarlo G, Gottschall WP, Morgan D, Arendash GW. Correlation between cognitive deficits and Abeta deposits in transgenic APP+PS1 mice. Neurobiol Aging. 2001;22:377-385.

6. Guo LL, Guan ZZ, Huang Y, Wang YL, Shi JS. The neurotoxicity of $\beta$-amyloid peptide toward rat brain is associated with enhanced oxidative stress, inflammation and apoptosis, all of which can be attenuated by scutellarin. Exp Toxicol Pathol. 2013;65:579-584.

7. Cynis H, Frost JL, Crehan H, Lemere CA. Immunotherapy targeting pyroglutamate-3 A $\beta$ : prospects and challenges. Mol Neurodegener. 2016;11:48.

8. Tewari D, Stankiewicz AM, Mocan A, Sah AN, Tzvetkov NT, Huminiecki L, Horbańczuk JO, Atanasov AG. Ethnopharmacological approaches for dementia therapy and significance of natural products and herbal drugs. Front Aging Neurosci. 2018;10:3.

9. Liu YX, Huang HC, Chang P, Jiang ZF. Mechanisms of neuroprotective activities of catechins in Alzheimer's disease. Nat Prod Res Dev. 2013;(11):1607-1613.

10. Hamaguchi T, Ono K, Yamada M. REVIEW: Curcumin and Alzheimer's disease. CNS Neurosci Ther. 2010;16:285-297.

11. Benesch MG, McElhaney RN. A comparative calorimetric study of the effects of cholesterol and the plant sterols campesterol and brassicasterol on the thermotropic phase behavior of dipalmitoylphosphatidylcholine bilayer membranes. Biochim Biophys Acta. 2014;1838:1941-1949.

12. Baskar AA, Al Numair KS, Gabriel Paulraj M, Alsaif MA, Muamar MA, Ignacimuthu S. $\beta$-sitosterol prevents lipid peroxidation and improves antioxidant status and histoarchitecture in rats with 1,2-dimethylhydrazine-induced colon cancer. J Med Food. 2012;15:335343.

13. Burg VK, Grimm HS, Rothhaar TL, Grösgen S, Hundsdörfer B, Haupenthal VJ, Zimmer VC, Mett J, Weingärtner O, Laufs U, Broersen LM, Tanila H, Vanmierlo T, Lütjohann D, Hartmann T, Grimm MO. Plant sterols the better cholesterol in Alzheimer's disease? A mechanistical study. J Neurosci. 2013;33:16072-16087.

14. Wang J, Wu F, Shi C. Substitution of membrane cholesterol with $\beta$-sitosterol promotes nonamyloidogenic cleavage of endogenous amyloid precursor protein. Neuroscience. 2013;247:227-233.

15. Garcia-Alloza M, Robbins EM, Zhang-Nunes SX, Purcell SM, Betensky RA, Raju S, Prada C, Greenberg SM, Bacskai BJ, Frosch MP. Characterization of amyloid deposition in the APPswe/PS1dE9 mouse model of Alzheimer disease. Neurobiol Dis. 2006;24:516-
524.

16. Sadowski M, Pankiewicz J, Scholtzova H, Ji Y, Quartermain D, Jensen CH, Duff K, Nixon RA, Gruen RJ, Wisniewski T. Amyloidbeta deposition is associated with decreased hippocampal glucose metabolism and spatial memory impairment in APP/PS1 mice. $J$ Neuropathol Exp Neurol. 2004;63:418-428.

17. Stavnezer AJ, Hyde LA, Bimonte HA, Armstrong CM, Denenberg VH. Differential learning strategies in spatial and nonspatial versions of the Morris water maze in the C57BL/6J inbred mouse strain. Behav Brain Res. 2002;133:261-270.

18. Folci A, Murru L, Vezzoli E, Ponzoni L, Gerosa L, Moretto E, Longo F, Zapata J, Braida D, Pistillo F, Bähler M, Francolini M, Sala M, Bassani S. Myosin IXa binds AMPAR and regulates synaptic structure, LTP, and cognitive function. Front Mol Neurosci. 2016;9:1.

19. Elliott E, Laufer O, Ginzburg I. BAG-1M is up-regulated in hippocampus of Alzheimer's disease patients and associates with tau and APP proteins. J Neurochem. 2009;109:1168-1178.

20. Takata K, Kitamura Y, Taniguchi T. Pathological changes induced by amyloid- $\beta$ in Alzheimer's disease. Yakugaku Zasshi. 2011;131:311.

21. Maier M, Peng Y, Jiang L, Seabrook TJ, Carroll MC, Lemere CA. Complement $\mathrm{C} 3$ deficiency leads to accelerated amyloid beta plaque deposition and neurodegeneration and modulation of the microglia/macrophage phenotype in amyloid precursor protein transgenic mice. J Neurosci. 2008;28:6333-6341.

22. Saeed AA, Genové G, Li T, Hülshorst F, Betsholtz C, Björkhem I, Lütjohann D. Increased flux of the plant sterols campesterol and sitosterol across a disrupted blood brain barrier. Steroids. 2015;99(Pt B):183-188.

23. Shi C, Liu J, Wu F, Zhu X, Yew DT, Xu J. $\beta$-sitosterol inhibits high cholesterol-induced platelet $\beta$-amyloid release. J Bioenerg Biomembr. 2011;43:691-697.

24. Zhang MY, Zheng CY, Zou MM, Zhu JW, Zhang Y, Wang J, Liu CF, Li QF, Xiao ZC, Li S, Ma QH, Xu RX. Lamotrigine attenuates deficits in synaptic plasticity and accumulation of amyloid plaques in APP/PS1 transgenic mice. Neurobiol Aging. 2014;35:2713-2725.

25. Guénette SY. Mechanisms of Abeta clearance and catabolism. Neuromolecular Med. 2003;4:147-160.

26. Westerman MA, Cooper-Blacketer D, Mariash A, Kotilinek L, Kawarabayashi T, Younkin LH, Carlson GA, Younkin SG, Ashe $\mathrm{KH}$. The relationship between Abeta and memory in the Tg2576 mouse model of Alzheimer's disease. J Neurosci. 2002;22:1858-1867.

27. Zhang RS, Xu HJ, Jiang JH, Han RW, Chang M, Peng YL, Wang Y, Wang R. Endomorphin-1 attenuates A $\beta 42$ induced impairment of novel object and object location recognition tasks in mice. Brain Res. 2015;1629:210-220.

28. Holtzman DM, Zlokovic B. Role of A $\beta$ transport and clearance in the pathogenesis and treatment of Alzheimer's disease. In: Sisodia SS, Tanzi RE, editors. Alzheimer's disease. Boston, MA: Springer; 2007. p. 179-198.

29. Li W, Yu J, Liu Y, Huang X, Abumaria N, Zhu Y, Huang X, Xiong W, Ren C, Liu XG, Chui D, Liu G. Elevation of brain magnesium prevents synaptic loss and reverses cognitive deficits in Alzheimer's disease mouse model. Mol Brain. 2014;7:65.

30. Ingelsson M, Fukumoto H, Newell KL, Growdon JH, Hedley-Whyte ET, Frosch MP, Albert MS, Hyman BT, Irizarry MC. Early Abeta accumulation and progressive synaptic loss, gliosis, and tangle for- 
mation in AD brain. Neurology. 2004;62:925-931.

31. Perez-Cruz C, Nolte MW, van Gaalen MM, Rustay NR, Termont A, Tanghe A, Kirchhoff F, Ebert U. Reduced spine density in specific regions of CA1 pyramidal neurons in two transgenic mouse models of Alzheimer's disease. J Neurosci. 2011;31:3926-3934.

32. Duffy FH, Albert MS, McAnulty G. Brain electrical activity in patients with presenile and senile dementia of the Alzheimer type. Ann Neurol. 1984;16:439-448. 